\title{
Quadrifilar Helix Antenna for GPS Applications
}

\author{
Cheng-Wei Lan and Jean-Fu Kiang \\ Department of Eelectrical Engineering and \\ Graduate Institute of Communication Engineering \\ National Taiwan University \\ Taipei, Taiwan, ROC \\ E-mail : jfkiang@cc.ee.ntu.edu.tw
}

\begin{abstract}
In GPS applications, the antenna requires omnidirectional pattern over approximately the entire upper hemisphere where the satellites may be visible. The fractional $Q H A(R Q H A)$ is a compact size antenna which is suitable for GPS application which mobile handset.In this paper, we will present a FQHA design with square cross section.
\end{abstract}

Keywords: helix, k- $\beta$ diagram, back-fire mode, GPS, axial ratio, beamwidth, QHA,

\section{Introduction}

Quadrifilar helical antenna is one of the most commonly used antenna for satellite communications. The QHA produces a cardioid-shaped radiation pattern with excellent circular polarization over wide angular range. Furthermore, fractional-turn QHA is a compact size antenna which is suitable in mobile handset for global positioning system(GPS) applications. The effect of dielectric core on traveling-wave QHA will be presented. Furthermore, resonant QHA with dielectric core will also be given. Dielectric loading is also applied to reduce antenna size. Moreover, square dielectric core is used to obtain broader bandwidth than cylindrical dielectric core.

\section{Traveling-Wave Quadrifilar Helical Antenna (QHA)}

A quadrifilar helical antenna can support both traveling-wave type and resonant type of operation. The element length decides the type of operation. This section discusses the traveling-wave type of operation.

\subsection{QHA and Unifilar Helix Antenna}

Operation of a QHA is similar in some respects to an unifilar helix antenna. The determinantal equation of $\mathrm{N}$ filar tape helix, excited in the $\exp (-\mathrm{j} \phi)$ mode, becomes identical to the sheath helix as the number, $\mathrm{N}$, becomes infinite [2]. In this way, we can estimate the properties of traveling-wave type QHA by the theory of unifilar helix.

Periodic structure approach can be applied to analyze the helix of infinite extent.

Fig.1 shows a three-dimensional helix structure which may be either left or right handed. Parameters to describe a helix structure include helix diameter(D), helix radius(a) circumference(C), spacing between turns(S), pitch angle $(\alpha)$, length of turn(L), and number of turns(N).

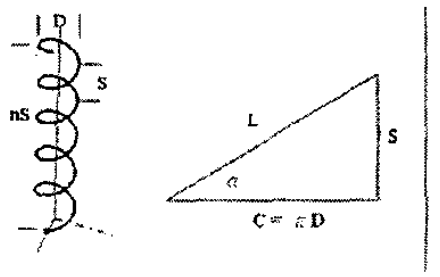

Fig1. Helix structure.

\section{$2.2 \quad k-\beta$ Diagram of Helix structure}

Periodic structure approach can be applied to analyze the helix of infinite extent by the mode diagram shown in Fig2.

Three modes of operation are marked as modes $a, b$ c, representing the normal, scanning(back-fire), and axial modes, respectively.

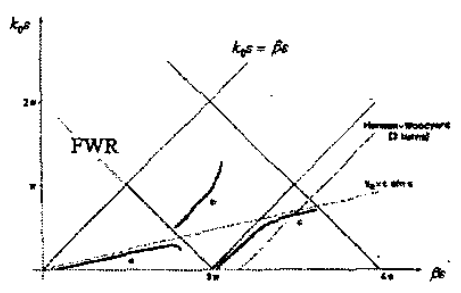

Fig2. k- $\beta$ diagram of helix structure [2]. 
In curve $a$, the cylindrical harmonic of $m=0$ dominates. The axial mode is dominated by the righthanded cylindrical harmonic of $m=-1$, also referred to as the $m=-1$ super-mode.

\subsection{Estimation QHA Using $\boldsymbol{k}-\boldsymbol{\beta}$ Diagram}

Fig. 3 shows the current distribution of a travelingwave QHA and its radiation pattern associated with the back-fire mode( $b$ mode) in the $k-\beta$ diagram.

Fig.3(a) shows the phase progression of current which propagates at the speed of light in free space. Fig.3.(b) shows the current amplitude which indicates a leaky-current. Fig3(c) shows the back-fire radiation pattern. The $b$ mode is a leaky-wave and back-fire radiation pattern. As the frequency increases, the beam scans from back-fire to broadside. Fig.4. shows that for the same QHA, the beam bends towards the broadside direction with increasing operation frequency.

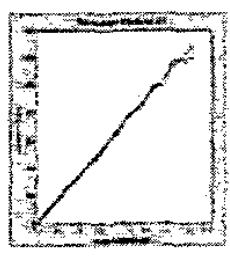

(a). phase

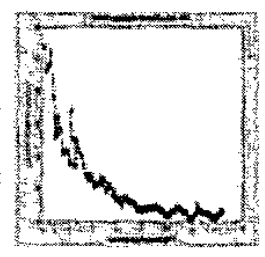

(b). amplitude

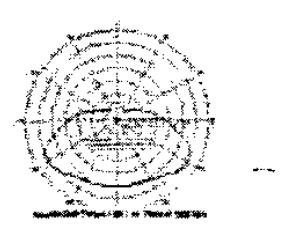

(c) pattern
Fig3. current distribution of traveling-wave QHA.

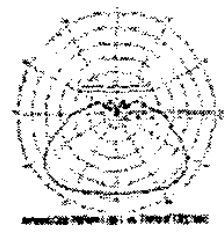

(a) $1.3 \mathrm{GHz}$

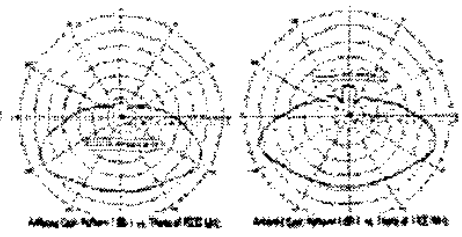

(c). $1.7 \mathrm{GHz}$

(b). $1.5 \mathrm{GHz}$ (c). $1.7 \mathrm{GH}$

Fig4. Radiation pattern of QHA

\subsection{Traveling-Wave QHA with Dielectric Core}

Fig.5 shows the structure of a traveling-wave QHA. The pitch angle is $49^{\circ}$, the circumference is $94.2 \mathrm{~mm}$, and the number of turn is 5 . The operation frequency is $1.5 \mathrm{GHz}$. Four tape helices are fed with equal-amplitude signal with relative phases of $0,90,180$, and $270 \mathrm{deg}$., respectively.

The dielectric core has the effect of slowing the current along the helical conductor. On the $k-\beta$ diagram, the slower current velocity means that the slope of the uncoupled mode curve is decreased with associated decrease in bandwidth for a helix with the same pitch angle. An infinitely long QHA exhibits the same behavior.

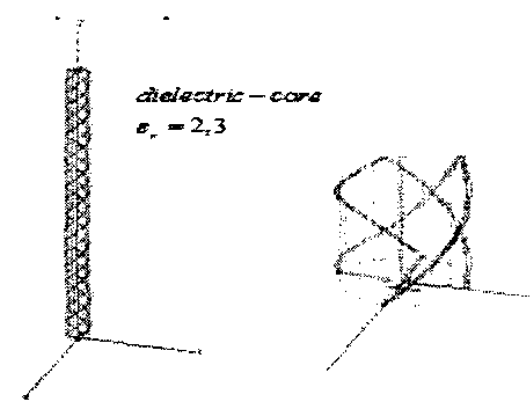

Fig5.QHA with dielectric core.

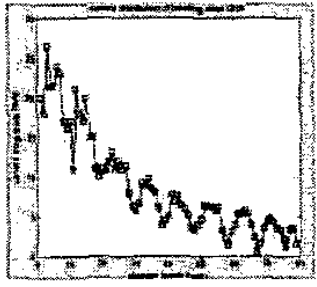

(a) $\varepsilon_{r}=2$

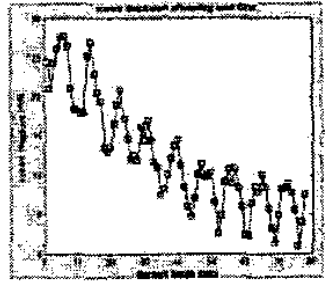

(b) $\varepsilon_{r}=3$
Fig.6 Current distribution of QHA with dielectric core.

Fig.6. shows the effect of dielectric core on travelingwave QHA. The dielectric core increases the reflected wave from its open end. Fig.6.(a). shows the current distribution of QHA with dielectric core of $\varepsilon_{\mathrm{r}}=2$. The reflected wave becomes stronger when the dielectric constant is increased. The standing wave ratio will decrease when helix length is increased.

\section{Quadrifilar Helical Antenna of Resonant Type}

\subsection{Fractional-Turn Quadrifilar Helical Antenna}

In GPS applications, the antenna requires omnidirectional pattern over approximately the entire upper hemisphere where the satellites may be visible. The fractional QHA(RQHA) is a compact size antenna which is suitable for GPS applications embedded in mobile handsets.

Thought RQHA is a compact size antenna, mobile nandset for satellite communication applications demands smaller antenna. Using of dielectric loading for RQHA 
can reduce the antenna size. However, there is a disadvantage with dielectric loading, the antenna has a low gain or poor radiation efficincy.

In this paper, we present a new square resonant quadrifilar helical antenna(SRQHA). The simulation results show fairly good circular polarization, radiation pattern, return loss bandwidth, and wide beamwidth. The radiation efficiency is higher than cylindrical RQHA.

\subsection{Resonant QHA with Dielectric Core}

Fig.7 shows the current distribution of RQHA. The current indicates that a short $\mathrm{QHA}$ will support a resonant mode. This type of antennas has the properties of small size and narrow bandwidth comparing with travelingwave QHA. However, using dielectric core for resonant QHA will have fairly good performances of radiation pattern, beamwidth, axial ratio, F/B ratio. However, the dielectric core will degrade the antenna efficiency.

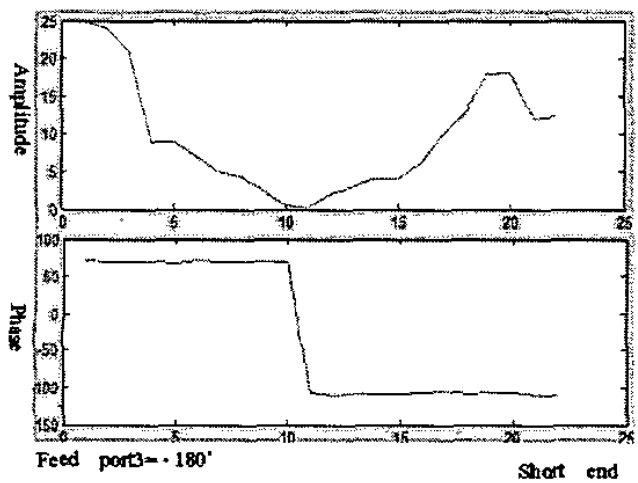

Fig7. Current distribution of RQHA

RQHA with dielectric core behaves like a dielectric resonator. In order to increase its radiation efficiency, we may increase the surface area of an RQHA. Hence, cylindrical core is replaced by rectangular core to extend its surface area with the same volume.

\subsection{Square Resonant QHA with dielectric core}

Fig7 shows a square resonant QHA(SRQHA) with dielectric core of $\varepsilon_{\mathrm{r}}=5$. The size of SRQHA is $14.4 \times 4.4$ $\times 35(\mathrm{~mm})$, which has a size reduction of $45 \%$ compared to an air core which is $32 \times 32 \times 64(\mathrm{~mm})$. The resonant frequency is $1.565 \mathrm{GHz}$ (GPS L1 frequency is $1.575 \mathrm{GHz}$ ). The antenna input impedance is $4 \Omega$.

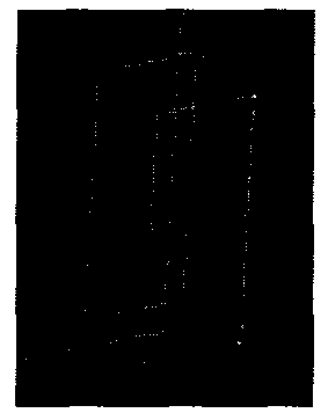

Fig8.structure of square resonant QHA with dielectric core.

Fig8. shows the radiation properties of the SRQHA. The $3 \mathrm{~dB}$ beamwidth is $124^{\circ}$, front-to-back ratio is $13 \mathrm{~dB}$, axial ratio is $0.34 \mathrm{~dB}$ at $\theta=0^{\circ}$, maximum value is $2.53 \mathrm{~dB}$.

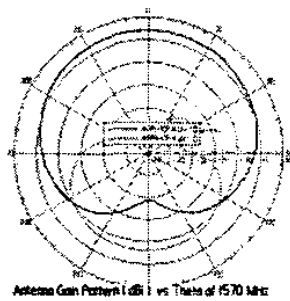

(a) gain pattern

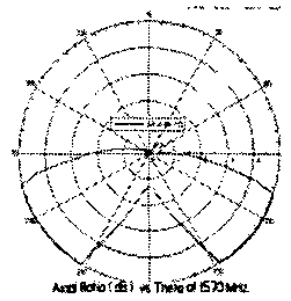

(b) axial ratio
Fig8. Square resonant QHA with dielectric core

Fig9. shows the return loss performance of SRQHA which has a $10 \mathrm{MHz}$ bandwidth $(-10 \mathrm{~dB}$ return loss).

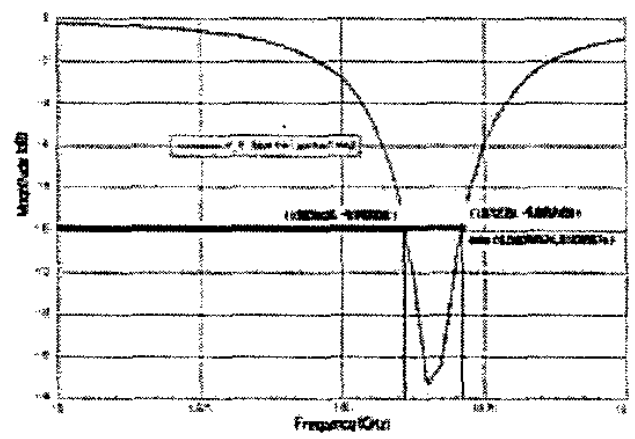

Fig9. Return loss of SRQHA 


\section{Conclusions}

Traveling-wave QHA with dielectric core will degrade the performance of radiation pattern and axial ratio. Using dielectric core for resonant QHA maintains the same performance in pattern and axial ratio. However, the size of antenna is reduced by $45 \%$.

\section{References}

[1] Rodney.G. Vaughan, J.Bach. Andersen, "Polarization Properties of the Axial Mode Helix Antenna," IEEE Trans on Antennas and Propagation ,vol-Ap33,no.1,January 1985.

[2] R.M. Barts and W.L. Stutxman, "A reduced size helical antenna," Antenna Laboratory, Virginia Tech.

[3] Jalil Rashed and Chen-To Tai, "A new class of resonant antennas", IEEE Trans. on Antennas and Propagation, vol 39,no9,September 1991.

[4] A,R Neureuther, P.Wklock, and R.Mittra, "A study of Sheath Helix with a Conducting Core and its Application to the Helical Antenna", IEEE Trans. on Antennas and Propagation, vol AP-15,no2,March 1967.

[5] C. C. Kilgus, "Resonant Quadrafilar Helix," IEEE Trans. Antenna and Propagation, Vol. Ap-17, May 1969 pp.349 351

[6] C. C. Kilgus, "Shaped-conical radiation pattern performance of the backfire quadrifilar helix," IEEE Trans. Antenna and Propagation, Vol. Ap-23 May 1975 pp.392 397 